\title{
Axion-Higgs unification
}

\author{
Michele Redi ${ }^{a, b}$ and Alessandro Strumia ${ }^{c, d}$ \\ ${ }^{a}$ CERN, Theory Division, \\ CH-1211, Geneva 23, Switzerland \\ ${ }^{b} I N F N$, \\ 50019 Sesto Fiorentino, Firenze, Italy \\ ${ }^{c}$ Dipartimento di Fisica dell'Università di Pisa and INFN, \\ Pisa, Italy \\ ${ }^{d}$ National Institute of Chemical Physics and Biophysics, \\ Tallinn, Estonia \\ E-mail: michele.redi@cern.ch, alessandro.strumia@cern.ch
}

ABSTRACT: In theories with no fundamental scalars, one gauge group can become strong at a large scale $\Lambda$ and spontaneously break a global symmetry, producing the Higgs and the axion as composite pseudo-Nambu-Goldstone bosons. We show how KSVZ and DFSZ axion models can be naturally realised. The assumption $\Lambda \approx 10^{11} \mathrm{GeV}$ is phenomenologically favoured because: a) The axion solves the QCD $\theta$ problem and provides the observed DM abundance; b) The observed Higgs mass is generated via RGE effects from a small Higgs quartic coupling at the compositeness scale, provided that the Higgs mass term is fine-tuned to be of electroweak size; c) Lepton, quark as well as neutrino masses can be obtained from four-fermion operators at the compositeness scale. d) Extra fermions can unify the gauge couplings.

KEywords: Technicolor and Composite Models, CP violation

ARXIV EPRINT: 1208.6013 


\section{Contents}

1 Introduction 1

2 Models with composite Higgs and KSVZ axion 3

2.1 A simple model 4

3 Models with composite Higgs and DFSZ axion $\quad 7$

$\begin{array}{lll}3.1 & \text { A simple model } & 7\end{array}$

4 Partially composite axion-Higgs $\quad 8$

5 The Higgs mass $\quad 10$

5.1 The gauge contribution 11

$\begin{array}{ll}5.2 \text { The fermion contribution and tuning } & 12\end{array}$

6 Conclusions and future directions 13

$\begin{array}{lr}\text { A Structure of the potential } & \mathbf{1 4}\end{array}$

A.1 Potential in the $\mathrm{SU}(6)_{L} \otimes \mathrm{SU}(6)_{R} / \mathrm{SU}(6)_{L+R}$ model 14

$\begin{array}{lll}\text { A.2 Potential in the } \mathrm{SU}(6) / \mathrm{SO}(6) \text { model } & 15\end{array}$

\section{Introduction}

Present flavour data, precision data, LHC data are compatible with the Standard Model (SM) and no new physics. We will assume that this means that the naturalness criterion adopted as guideline by many theorists in the past 30 years is wrong.

Furthermore, the measured Higgs mass $m_{h}=125.5 \pm 0.5 \mathrm{GeV}[1,2]$ corresponds to a Higgs quartic coupling $\lambda$ close to zero when renormalized at energies above $\Lambda \approx$ $10^{11} \mathrm{GeV}$ [3]. A small quartic $\lambda$ can have alternative theoretical interpretations in terms of new physics at the scale $\Lambda$, such as

1) Supersymmetry with $\tan \beta=1$ broken at $m_{\mathrm{SUSY}} \approx \Lambda[4-6]$;

2) The Higgs could be the 5-th component of a gauge field living in one extra dimension with radius $R \approx \Lambda^{-1}$;

3) The Higgs could be the Nambu-Goldstone-Boson (NGB) of a global symmetry $G$ spontaneously broken to a subgroup $H$ at some high scale $f \approx \Lambda$. 
We here explore the third possibility that finds a natural realization in theories where one extra 'Techni-Color' (TC) gauge interaction becomes strong at the scale $\Lambda$ [7-9]. ${ }^{1}$ We are interested in Composite Higgs $(\mathrm{CH})$ models $[11,12]$, where condensates break a global symmetry producing the Higgs as a pseudo-NGB, while the SM gauge group is not broken by the strong dynamics. One general feature is a small Higgs quartic coupling at the TC scale. The quartic coupling responsible for the observed Higgs mass is then generated dominantly via SM RGE running down to the weak scale.

Composite Higgs models, already discussed at the weak scale $\Lambda \sim \mathrm{TeV}$ as possible solutions to the hierarchy problem, have various problems: no new physics has so far shown up in experiments such that one needs to resort to ad-hoc constructions with no known UV completion. All these problems disappear if $\Lambda \sim 10^{11} \mathrm{GeV}$, and furthermore RGE corrections enhanced by $\ln \Lambda / v$ generate the Higgs potential even starting from $V(h)=0$ at tree level. One has to accept a huge fine-tuning of the electro-weak scale $(\Lambda / v)^{2} \sim 10^{18}$, possibly explained by 'anthropic selection' [13].

Another motivation for this work is that TC models tend to automatically predict axion candidates [7-9]. If $\Lambda \sim \mathrm{TeV}$ this is an embarrassment — weak scale axions are excluded by experiments, while Dark Matter considerations favour a much higher axion scale, $f_{a} \approx 10^{11} \mathrm{GeV}[14-16]$ - so that appropriate mechanisms must be invoked to remove weak-scale axions. The natural occurrence of axions becomes a virtue in our scenario where the TC scale is around $10^{11} \mathrm{GeV}$ : such models can simultaneously explain the QCD $\theta$ problem and Dark Matter and lead to predictions for the axion/photon couplings.

Furthermore, if these models could be extended into models for fermion masses following the old idea of Extended Technicolor (ETC) [7-9], neutrino masses can naturally arise with the observed order of magnitude, $m_{\nu} \sim v^{2} / \Lambda \sim \mathrm{eV}$.

In this paper we do not attempt such ambitious ETC construction, and explore models where the axion and Higgs are unified in an irreducible coset $G / H$. They can be divided in two categories, depending on how the QCD anomaly of the axion symmetry is generated:

A) from new fermions (KSVZ axions). Within models where the Higgs and axion are unified in a single coset $G / H$ this possibility arises when the full SM gauge group is contained in the unbroken group $H$. This can realize KSVZ axions [17, 18].

B) from the SM fermions (DFSZ axions). This possibility arises when color $\mathrm{SU}(3)_{c}$ is not contained in the unbroken group $H$. Since there is no contribution to the QCD anomaly from TC fermions the anomaly must be generated by the SM fermions. The composite sector can however contribute to the electro-magnetic anomaly, because the unbroken group $H$ contains the electro-weak group. This is similar to DFSZ axions $[19,20]$.

Another distinction arises because, within each class, the SM fermion masses can either be generated:

\footnotetext{
${ }^{1}$ Other realizations of the same idea are in weakly coupled theories, or in models with extra dimensions (warped or not warped) where the Higgs is the Wilson line of an extra-dimensional gauge field [10].
} 
a) from bi-linear couplings of SM fermions to TC fermions. This possibility can be presumably realized in appropriate ETC constructions that unify the SM gauge group and TC interactions.

b) from linear couplings of SM fermions to fermions of the TC sector. This possibility, known as partial compositeness, is often considered in recent attempts of building weak-scale composite Higgs models compatible with data, but it seems harder to realize this in known UV completions.

KSVZ models are explored in section 2; DSFZ models in section 3; models with partial compositeness in section 4 . The Higgs mass in models with high compositeness scale is discussed in section 5. In section 6 we conclude. Technical details and estimates of the Higgs potential are collected in the appendix.

\section{Models with composite Higgs and KSVZ axion}

First, let us recall that KSVZ axion models $[17,18]$ add to the SM vector-like heavy quarks $\Psi_{Q}=\left(Q_{L}, \bar{Q}_{R}\right)^{2}$ and a heavy complex scalar $\sigma$ with a Peccei-Quinn (PQ) symmetry

$$
\Psi_{Q} \rightarrow e^{i \alpha_{Q}} \Psi_{Q}, \quad \sigma \rightarrow e^{-2 i \alpha_{Q}} \sigma
$$

that forbids the $\Psi_{Q}$ mass term. Heavy quark masses only come from the VEV of $\sigma$ that spontaneously breaks the chiral symmetry:

$$
\mathscr{L}=\mathscr{L}_{\mathrm{SM}}+\bar{\Psi}_{Q} i \not \partial \Psi_{Q}+\left|\partial_{\mu} \sigma\right|^{2}+\left(\lambda \sigma \bar{\Psi}_{Q} \Psi_{Q}+\text { h.c. }\right)-V(\sigma)
$$

giving rise to a light axion $a=\sqrt{2} \operatorname{Im} \sigma$ with large decay constant $f_{a} \approx\langle\sigma\rangle$.

Composite Higgs models that realize the KSVZ axion can be broadly characterised as follows. The broken generators of $G / H$ contain the PQ symmetry; the unbroken group $H$ contains the SM gauge group and the NGB include the Higgs doublet and the axion singlet. To solve the strong CP problem the axionic symmetry should be anomalous under QCD but not under TC gauge interactions.

Given that the SM gauge group is a subset of SU(5), we find that a minimal possibility is provided by ${ }^{3}$

$$
\frac{G}{H}=\frac{\mathrm{SU}(6)_{L} \times \mathrm{SU}(6)_{R}}{\mathrm{SU}(6)_{L+R}} .
$$

The 35 NGBs decompose under $\mathrm{SU}(5)_{\mathrm{SM}}$ as $\mathbf{2 4} \oplus \mathbf{5} \oplus \overline{\mathbf{5}} \oplus \mathbf{1}$. The 33 scalars charged under the SM interactions typically acquire a large mass of order $g_{\mathrm{SM}} \Lambda / 4 \pi$. We assume that the scalar with the quantum numbers of the Higgs boson remains accidentally light presumably due to 'anthropic cancellations' in the potential, see section 5. The two scalar SM singlets are axion candidates.

\footnotetext{
${ }^{2}$ Here and in what follows all fermions are two-components left-handed Weyl fermions.

${ }^{3} \mathrm{SU}(5)_{L} \times \mathrm{SU}(5)_{R} / \mathrm{SU}(5)_{L+R}$ would not work because the NGBs do not include doublets.
} 


\section{$2.1 \quad$ A simple model}

To be concrete, the above scenario can be realized adding to the $\mathrm{SM}$ an $\mathrm{SU}(n)$ TC group and massless TC-fermions transforming in the following representations of the full gauge group, $\mathrm{U}(1)_{Y} \otimes \mathrm{SU}(2)_{L} \otimes \mathrm{SU}(3)_{c} \otimes \mathrm{SU}(n)_{\mathrm{TC}}$ :

\begin{tabular}{|c|c|c|c|c|}
\hline Fermion & $\mathrm{U}(1)_{Y}$ & ${ }_{V} \mathrm{SU}(2)_{L}$ & $L \mathrm{SU}(3)_{\mathrm{c}}$ & $\mathrm{U}(n)_{\mathrm{TC}}$ \\
\hline$D$ & $\frac{1}{2}$ & 1 & $\overline{3}$ & $n$ \\
\hline$L$ & $-\frac{1}{2}$ & 2 & 1 & $n$ \\
\hline$N$ & 0 & 1 & 1 & $n$ \\
\hline $\bar{D}$ & $-\frac{1}{3}$ & 1 & 3 & $\bar{n}$ \\
\hline $\bar{L}$ & $\begin{array}{l}3 \\
\frac{1}{2}\end{array}$ & $\overline{2}$ & 1 & $\bar{n}$ \\
\hline $\bar{N}$ & 0 & 1 & 1 & $\bar{n}$ \\
\hline
\end{tabular}

Massless TC fermions can be enforced with a $\mathrm{Z}_{2}$ symmetry. Notice that we do not make use of any fundamental scalar: $D, L$ and $N$ are TC-colored copies of the SM fermions (right-handed down quarks $d$, left-handed lepton doublet $\ell$, right-handed neutrino $n$ ), and they fill full SU(5) multiplets $\overline{5} \oplus 1$. The main difference with respect to TC constructions is the addition of fermions $\bar{D}, \bar{L}$ and $\bar{N}$ in the conjugated representations, such that TC fermions are vectorial with respect to the SM gauge group. This guarantees that the TC dynamics does not break the electro-weak symmetry. Indeed the following condensates develop

$$
\langle D \bar{D}\rangle=\langle L \bar{L}\rangle=\langle N \bar{N}\rangle \approx \Lambda^{3}
$$

realizing the pattern (2.3) of symmetry breaking. Among the 36 scalars $D \bar{D}, L \bar{L}, N \bar{N}$, $D \bar{L}, L \bar{D}, D \bar{N}, N \bar{D}, L \bar{N}, N \bar{L}$ there is a single NGB Higgs doublet that corresponds to the combination

$$
H \sim(L \bar{N})-(\bar{L} N)^{*}
$$

and three neutral real states: $D \bar{D}, L \bar{L}$ and $N \bar{N}$. One of them, $D \bar{D}+L \bar{L}+N \bar{N}$ is not a true NGB: it gets a mass of order $g_{T C} \Lambda / 4 \pi$ from the $\mathrm{U}(1)_{\mathrm{PQ}} \times \mathrm{SU}(n)_{\mathrm{TC}}^{2}$ anomaly, just like the $\eta^{\prime}$ in QCD. The orthogonal combinations typically have a QCD anomaly and provide viable axion candidates.

Their precise phenomenology depends on model options not specified so far. Let us summarize the main possibilities.

1. If high-scale dynamics respects their global symmetries, at low energy one has two light NGB associated to the U(1) charges,

$$
\frac{4 D-3 L-6 N}{\sqrt{102}}, \quad \frac{L-2 N}{\sqrt{3}} .
$$

The first one has a QCD anomaly and behaves as an axion with electromagnetic coupling $E / N=-5 / 6{ }^{4}$ The presence of the other NGB does not pose a phenomenological problem, because its interactions are suppressed by $f \sim 10^{11} \mathrm{GeV}[22]$.

\footnotetext{
${ }^{4}$ We recall that it is defined as $E / N=\sum Q_{\mathrm{PQ}} Q_{\mathrm{em}}^{2} / \sum Q_{\mathrm{PQ}} T^{2}$ where the sums extend over all fermions with PQ charges $Q_{\mathrm{PQ}}$, electric charges $Q_{\mathrm{em}}$, and QCD generators $\operatorname{Tr} T^{a} T^{b}=\frac{1}{2} T^{2} \delta^{a b}$. The electromagnetic
} 
2. If instead the $\mathrm{SM}$ gauge group is unified into $\mathrm{SU}(5)$ at a scale $M_{\mathrm{GUT}}$, then $D$ and $L$ merge into a $\overline{5}$ such that their phases cannot be independently rotated any more, and one of the two NGB gets a mass of order $\Lambda^{2} / M_{\text {GUT }}$. The NGB that survives at low energy and plays the role of the axion is the $\mathrm{SU}(5)$-invariant

$$
\frac{D+L-5 N}{\sqrt{30}}
$$

with $E / N=8 / 3 .^{5}$

3. SM Yukawa couplings presumably originate from 4-fermion operators, which could break none, some or all of the axion-like symmetries. (The latter option is the one pursued in weak scale TC models). For example, the Yukawa couplings for the SM up-type quarks $q, u$ can be generated by two kind of operators:

$$
(q u)(L \bar{N}), \quad(\bar{q} \bar{u})(\bar{L} N)
$$

If both of them are present, then one NGB gets a large mass of order $\approx y_{t} \Lambda$ and the surviving light axion is the combination under which the Higgs doublet is neutral:

$$
\frac{D-3 L+3 N}{\sqrt{30}}
$$

In such a case, the model predicts the experimentally observable ratio of axion electromagnetic and QCD anomalies to be $E / N=-16 / 3$.

Models where the only light composite particle is one axion (but not the Higgs) can be easily built along the same lines, overcoming the difficulties in [23].

Of course, one can build variants of the model with different TC-fermion content. For example the TC fermions might fill a full family multiplet $(\overline{\mathbf{5}} \oplus \mathbf{1 0} \oplus \mathbf{1}, \boldsymbol{n})+$ h.c. The global symmetry is now $G / H=\mathrm{SU}(16)_{L} \times \mathrm{SU}(16)_{R} / \mathrm{SU}(16)_{L+R}$. This is the composite Higgs analog of the one family TC model of Farhi-Susskind [24]. This model contains 7 axion candidates. The one coupled to $5 \cdot \mathbf{5}-2 \cdot \mathbf{1 0}-5 \cdot \mathbf{1}$ predicts $E / N=4 / 3$ and is particularly interesting, because it combines the good properties previously discussed: the Higgs has no PQ charge, and it is $\mathrm{SU}(5)$-invariant.

Alternatively, TC fermions might fill a 'tilted' family like $(\mathbf{5} \oplus \mathbf{1 0} \oplus \mathbf{1}, \boldsymbol{n})+$ h.c.: such models lead to extra states with the quantum numbers of the Higgs doublet (like $L E, Q D$ ).

coupling of the axion is predicted as

$$
\frac{g_{a \gamma \gamma}}{m_{a}}=\frac{\alpha_{\mathrm{em}}}{2 \pi f_{\pi} m_{\pi}} \sqrt{\left(1+\frac{m_{d}}{m_{u}}\right)\left(1+\frac{m_{u}}{m_{d}}+\frac{m_{u}}{m_{s}}\right)}\left[\frac{E}{N}-\frac{2}{3}\left(\frac{4+m_{u} / m_{d}+m_{u} / m_{s}}{1+m_{u} / m_{d}+m_{u} / m_{s}}\right)\right] .
$$

where $f_{\pi}=93 \mathrm{MeV}$. Thereby $E / N$ can be measured up to a two-fold ambiguity. Axion dark matter experiments such as ADMX [21] are reaching now the required sensitivity for the value of $f \approx 10^{11} \mathrm{GeV}$ suggested by the cosmological DM abundance.

${ }^{5}$ The fact that the Higgs doublet is charged under the symmetry associated to the axion is not a problem and does not affect their phenomenology, because one can compensate a change in the Higgs phase by a $\mathrm{U}(1)_{Y-B / 6}$ rotation of the SM fields. This transformation introduces no QCD nor QED anomalies. 

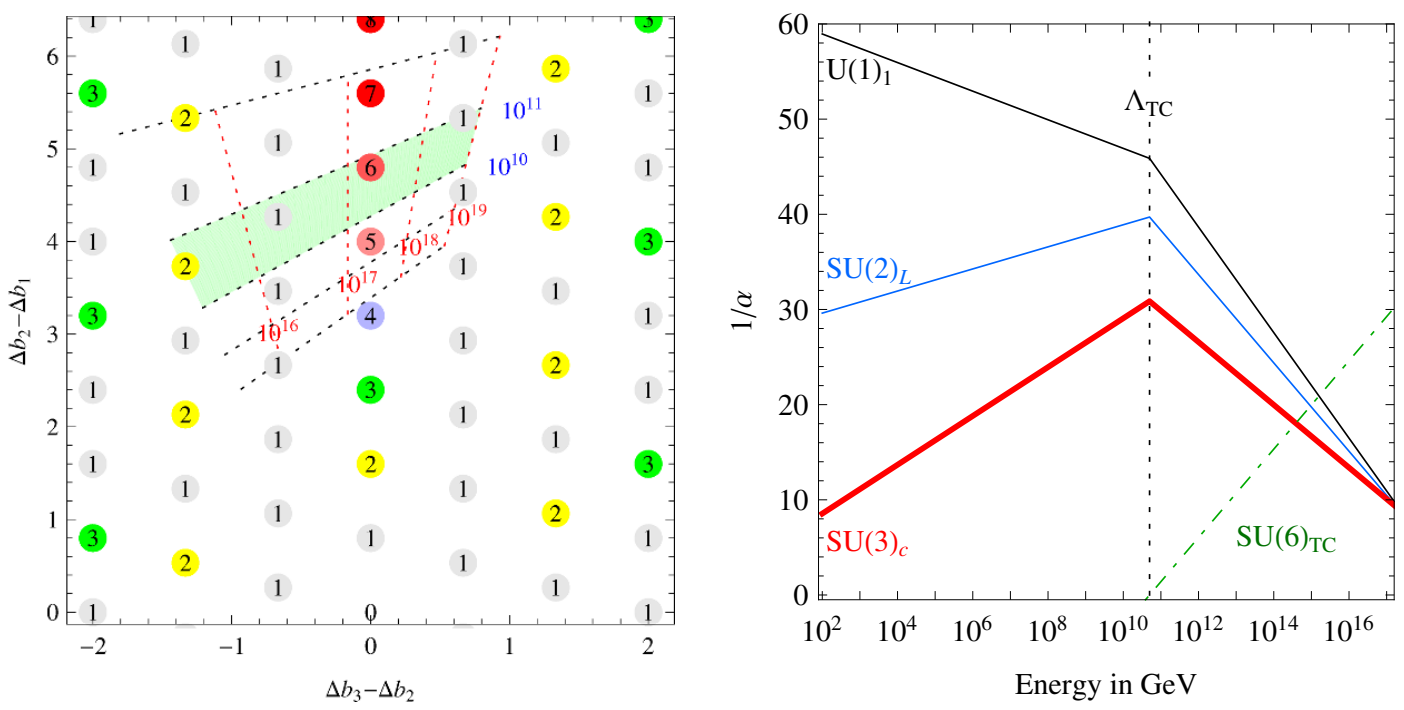

Figure 1. Left: possible values of the one-loop $\beta$-function coefficients generated by fermions in real representations of the SM group that can be embedded in SU(5) multiplets marked according to their maximal multiplicity (e.g. the point marked with 6 can be realised with up to 6 copies of the same set of fermions). The shading indicates the region favoured by unification with multiplets of mass $\Lambda$ between $10^{10}$ and $10^{11} \mathrm{GeV}$ (the GUT scale in $\mathrm{GeV}$ is indicated in red). Right: running of the gauge couplings (with hypercharge in GUT normalization) for one model that realises the point marked with 6 employing a $\mathrm{SU}(6)_{\mathrm{TC}} \mathrm{TC}$ interaction.

In the model above the presence of strong TC dynamics at $\Lambda \approx 10^{11} \mathrm{GeV}$ does not improve the unification of the SM gauge couplings, as the composite states come in complete multiplets. This remains true at any order (even non-perturbative) in the TC coupling.

So, one can instead consider TC fermions in incomplete $\mathrm{SU}(5)_{\text {GUT }}$ multiplets. Along the lines of [25], figure 1a shows the values of the one-loop $\beta$-function coefficients for the SM gauge couplings allowed by arbitrary combinations of fermions in real representations of the SM group that can be embedded in $\mathrm{SU}(5)_{\text {GUT }}$ multiplets. Within the shaded region, unification is achieved with an intermediate mass $\Lambda$ between $10^{11}$ and $10^{12} \mathrm{GeV} .{ }^{6}$ We added on each point a number $n$ equal to the maximal multiplicity of the fermions. This means that such point can be realised adding $n$ copies of the same set of fermions. This issue is relevant in the TC context, where TC fermions appear in the $\boldsymbol{n} \oplus \overline{\boldsymbol{n}}$ representation of a SU( $n)$ TC group. This singles out the points marked as $n=6$ and $n=5$ around the shaded region favoured by unification. Such points (where unification occurs at $M_{\mathrm{GUT}} \approx 2 \times 10^{17} \mathrm{GeV}$ ) can be obtained adding TC fermions in an incomplete SM family $D, L, Q, U, N$ (no righthanded leptons $E$ ) in the $\boldsymbol{n}$ representation of $\mathrm{SU}(n)_{\mathrm{TC}}$, plus their conjugated $\bar{D}, \bar{L}, \bar{Q}, \bar{U}, \bar{N}$ in the $\overline{\boldsymbol{n}}$. The corresponding evolution of the gauge couplings is shown in figure $1 \mathrm{~b}$ for the case $n=6$.

\footnotetext{
${ }^{6}$ Such region slightly differs from the analogous figure in [25] because in the present scenario the Higgs boson is not present above the compositeness scale.
} 


\section{Models with composite Higgs and DFSZ axion}

First, let us recall Dine-Fischler-Srednicki-Zhitnitskii (DFSZ) axion models [19, 20]. They use, instead of extra colored fermions, two Higgs doublets and one complex heavy neutral scalar $\sigma$. The theory is assumed to be symmetric under a global $\mathrm{U}(1)_{\mathrm{PQ}}$

$$
\sigma \rightarrow e^{4 i \alpha} \sigma, \quad q_{L, R} \rightarrow e^{i \alpha} q_{L, R} \quad H_{u} \rightarrow e^{-2 i \alpha} H_{u}, \quad H_{d} \rightarrow e^{-2 i \alpha} H_{d}
$$

such that a term $\sigma H_{u} H_{d}$ is allowed and present in the Lagrangian, while $H_{u} H_{d}$ and $\left(H_{u} H_{d}\right)^{2}$ terms are absent. The PQ symmetry is then broken by the vacuum expectation values of $H_{u}, H_{d}$ and $\sigma$. Assuming that $\sigma$ has a large vev $\langle\sigma\rangle \gg v$ the axion is $a=\sqrt{2} \operatorname{Im} \sigma$ with large decay constant $f_{a} \approx\langle\sigma\rangle$.

Composite Higgs models that realize this scenario can be characterised as follows. The group $G$ contains the PQ symmetry; the unbroken group $H$ contains the EW group (but not the QCD group), the NGB contain the axion and two Higgses, that must be charged under the PQ symmetry.

\subsection{A simple model}

In this section we describe a realization of this scenario based on the pattern

$$
\frac{G}{H}=\frac{\mathrm{SU}(6)}{\mathrm{SO}(6)}
$$

that generalizes the model of Kaplan and Georgi $[11,12]$ such that $\mathrm{SO}(6) \supset \mathrm{SO}(4) \otimes \mathrm{U}(1)_{\mathrm{PQ}}$, where $\mathrm{SO}(4) \supset \mathrm{SU}(2)_{L} \otimes \mathrm{SU}(2)_{R}$ contains the SM electroweak group (hypercharge is the $T_{3}$ generator of $\left.\mathrm{SU}(2)_{R}\right)$.

The pattern of symmetry breaking in eq. (3.2) can be obtained from a $\mathrm{SO}(n)_{\mathrm{TC}}$ gauge theory with 6 massless TC-fermions $\psi^{i}$ in the vectorial representation of the TC gauge

\begin{tabular}{|c|c|c|c|c|c|}
\hline Fermions & $\mathrm{U}(1)_{Y}$ & $\mathrm{SU}(2)_{L}$ & $\mathrm{SU}(3)_{\mathrm{c}}$ & $\mathrm{SO}(n)_{\mathrm{TC}}$ & $\mathrm{U}(1)_{\mathrm{PQ}}$ \\
\hline$L$ & $-\frac{1}{2}$ & 2 & 1 & $n$ & 0 \\
\hline $\bar{L}$ & $\frac{1}{2}$ & $\overline{2}$ & 1 & $n$ & 0 \\
\hline$N$ & 0 & 1 & 1 & $n$ & 2 \\
\hline $\bar{N}$ & 0 & 1 & 1 & $n$ & -2 \\
\hline
\end{tabular}
group [26]. They can be organized as

where $L=\left(\psi_{3}+i \psi_{4}, \psi_{1}-i \psi_{2}\right), \bar{L}=\left(\psi_{1}+i \psi_{2},-\psi_{3}+i \psi_{4}\right), N=\psi_{5}+i \psi_{6}$ and $\bar{N}=\psi_{5}-i \psi_{6}$ are the combinations with definite $\mathrm{PQ}$ charge.

The TC condensate $\langle L \bar{L}\rangle=\langle N \bar{N}\rangle=\Lambda^{3}$ breaks $\mathrm{SU}(6) \rightarrow \mathrm{SO}(6)$. The NGB, loosely written as

$$
L N, L \bar{N}, \bar{L} N, \bar{L} \bar{N} \oplus N N, \bar{N} \bar{N} \oplus N \bar{N} \oplus L L, L \bar{L}, \bar{L} \bar{L},
$$

transform as the $\mathbf{2 0}^{\prime}$ (symmetric traceless) representation of the unbroken $\mathrm{SO}(6)$, that decomposes under $\mathrm{SU}(2)_{L} \otimes \mathrm{SU}(2)_{R} \otimes \mathrm{U}(1)_{\mathrm{PQ}}$ as

$$
\mathbf{2 0}^{\prime}=(\mathbf{2}, \mathbf{2})_{ \pm \mathbf{2}} \oplus(\mathbf{1}, \mathbf{1})_{ \pm \mathbf{4}} \oplus(\mathbf{1}, \mathbf{1})_{\mathbf{0}} \oplus(\mathbf{3}, \mathbf{3})_{\mathbf{0}}
$$


where the subscripts show the PQ charge. The first two multiplets charged under $\mathrm{U}(1)_{\mathrm{PQ}}$ are the scalars $H_{u}, H_{d}$ and $\sigma$ of the DFSZ axion models; furthermore there is a singlet $\eta$ and scalars with EW gauge interactions that will acquire a large mass. We again assume that one Higgs doublet remains anthropically light.

The potential $V\left(\sigma, H_{u}, H_{d}\right)$ (which needs to have a minimum at $\sigma \approx \Lambda$ ) is generated only by interactions that break the global symmetry: in the minimal case these are gauge interactions and four-fermion operators needed to produce the SM Yukawas. We assume that such interactions respect the PQ symmetry, realizing a type-II two Higgs doublet model:

$$
\frac{1}{\Lambda_{t}^{2}}\left(q_{L} t_{R}^{c}\right)^{\dagger}(L N)+\frac{1}{\Lambda_{b}^{2}}\left(q_{L} b_{R}^{c}\right)^{\dagger}(\bar{L} \bar{N})+\text { h.c. }
$$

When converted into couplings to the NGBs $H_{u}$ and $H_{d}$, couplings to $H_{u}$ are accompanied by couplings to $\sigma^{*} H_{d}^{*} / f^{2}$, such that these interactions generate at one loop (see appendix A.2) the $\sigma H_{u} H_{d}$ term needed for the DFSZ axion mechanism.

The ratio $E / N$ is uniquely determined in these models. The electromagnetic anomaly of the TC sector is given by

$$
E_{\mathrm{TC}}=\operatorname{Tr}\left[Q_{\mathrm{PQ}} \cdot\left(T_{3 L}+T_{3 R}\right)^{2}\right]=2 n
$$

where $T_{3(L, R)}$ are $\mathrm{SO}(6)$ generators in the vector representation. The anomalies under the SM fields are identical to the model in $[19,20]$ and one finds,

$$
\frac{E}{N}=\frac{8}{3}+n
$$

If the strong sector is a large $n$ theory one could therefore obtain a large ratio $E / N$.

Let us briefly discuss neutrino masses. If the SM fields include the right-handed neutrino we can generate a small mass with the standard see-saw mechanism. Alternatively, compatibly with PQ symmetry, we can add the dimension 9 operator,

$$
\frac{1}{\Lambda_{\nu}^{4}}(\ell \bar{L})^{2} N^{2} \rightarrow \frac{1}{\Lambda_{\nu}^{3}}\left(\ell H_{u}\right)^{2} \sigma^{2}+\cdots
$$

that gives rise to Majorana masses of order $v^{2} / \Lambda$ after that $\sigma$ acquires a VEV of order $f$.

\section{Partially composite axion-Higgs}

In this section we discuss the most minimal model of Axion-Higgs unification, where the NGB are just the $4+1$ degrees of freedom of the Higgs doublet and of the axionic phase. Such scenario is realized by the coset

$$
\frac{G}{H}=\frac{\mathrm{SO}(6)}{\mathrm{SO}(5)} \simeq \frac{\mathrm{SU}(4)}{\mathrm{Sp}(4)}
$$

where $\mathrm{SO}(6)$ contains the EW gauge group and the PQ symmetry as $\mathrm{SO}(4) \otimes \mathrm{SO}(2)=$ $\mathrm{SU}(2)_{L} \otimes \mathrm{SU}(2)_{R} \otimes \mathrm{U}(1)_{\mathrm{PQ}}[27]$. The $5 \mathrm{NGBs}$, transforming in the 5 of $\mathrm{SO}(5)$, decompose into a doublet and a singlet under the electro-weak symmetry. The NGB can be 
parametrized with an $\mathrm{SO}(6)$ vector with unit norm that in the unitary gauge can be written in terms of the physical Higgs $h$ and axion $a$ as:

$$
\Phi=\left(0,0,0, \frac{h}{f}, \frac{a}{f}, \sqrt{1-\frac{h^{2}+a^{2}}{f^{2}}}\right) .
$$

The $\mathrm{U}(1)_{\mathrm{PQ}}$, which acts as rotation between the last two components of $\Phi$ is spontaneously broken by the strong dynamics at $\Lambda$ so that the EW singlet $a$ is the axion candidate. ${ }^{7}$

This symmetry breaking pattern can be realized with $\operatorname{Sp}(n)$ TC gauge interactions, and the following TC fermion content $[28,29]$

\begin{tabular}{|cccccc|}
\hline Fermions & $\mathrm{U}(1)_{Y}$ & $\mathrm{SU}(2)_{L}$ & $\mathrm{SU}(3)_{\mathrm{c}}$ & $\mathrm{Sp}(n)_{\mathrm{TC}}$ & $\mathrm{U}(1)_{\mathrm{PQ}}$ \\
\hline$D$ & 0 & 2 & 1 & $n$ & +1 \\
$S$ & $+\frac{1}{2}$ & 1 & 1 & $n$ & -1 \\
$\bar{S}$ & $-\frac{1}{2}$ & 1 & 1 & $n$ & -1 \\
\hline
\end{tabular}

Notice that $\operatorname{Sp}(n)$ dynamics induces a condensate that does not break $\mathrm{SU}(2)_{L}$, despite the presence of a single lepton doublet. From the $\mathrm{PQ}$ charges it follows that there are no TC-TC-PQ anomalies. At this level however $a$ is not a viable axion because QCDQCD-PQ anomalies are also absent: indeed when generating fermion masses by adding four-fermion operators like $(q u)(D S)$, the SM term and the TC term separately preserve the PQ symmetry. One way to remedy this is to generate flavor with dimension 9 operators of the form $(q u)(D S)(S \bar{S})$ so that the SM fields are charged under the PQ symmetry. Another way is to consider non minimal models adding extra TC fermions.

We here consider a different route and assume that fermion masses are generated through linear couplings to states of the strong sector. This hypothesis, known as Partial Compositeness, is for example realized in extra-dimensional theories, but as drawback it is not known a microscopic dynamics that realizes it. Within Partial Compositeness, SM fermions $\psi$ couple linearly to composite fermions $\Psi$, with identical quantum numbers under the SM group, effectively realizing the following structure:

$$
m \psi \Psi+M \Psi \Psi+g_{\mathrm{TC}} \Psi \Psi H .
$$

After integrating out the heavy fermions $\Psi$ one obtains Yukawa couplings $\left(m g_{\mathrm{TC}} / M\right) \psi \psi H$. Through the mixing terms the SM fields can become charged under the PQ symmetry generating in turn the required QCD anomaly.

As a simple example we consider the case where SM fermions couple to composite fermions $\Psi_{Q}, \Psi_{U}$ in the 6 of $\mathrm{SO}(6)$. For the purpose of writing invariants it is useful to promote the $\mathrm{SM}$ quarks to incomplete $\mathrm{SO}(6)$ multiplets. For the up sector,

$$
\begin{aligned}
\psi_{q} & =\frac{1}{\sqrt{2}}\left(b_{L},-i b_{L}, t_{L}, i t_{L}, 0,0\right), \\
\psi_{u} & =(0,0,0,0, i \cos \theta, \sin \theta) u_{R}
\end{aligned}
$$

\footnotetext{
${ }^{7}$ This was already noted in [27] where the same coset was considered. There however $f \sim v$ so that $\mathrm{U}(1)_{\mathrm{PQ}}$ had to be explicitly broken to remove the unwanted weak-scale axion. We take here the opposite point of view: $f$ is large so that the singlet plays the role of the QCD axion.
} 
and similarly for the other fermions, see $[27,30]$ for more details. The mixing of left-handed doublets $\psi_{q} \Psi_{Q}$ term is automatically invariant under $\mathrm{U}(1)_{\mathrm{PQ}}$, while the right-handed one $\psi_{u} \Psi_{U}$ is invariant provided that $\theta=\pi / 4$ and $u_{R}$ has unit charge. ${ }^{8}$

The one-loop potential for $\Phi$ is [30],

$$
V \simeq C_{1} \frac{h^{2}}{f^{2}}+C_{2} \frac{h^{2}}{f^{2}}\left[1-\frac{h^{2}}{f^{2}}\right]
$$

where the coefficients $C_{i} \sim g_{S M}^{2} \Lambda^{2} f^{2} /(4 \pi)^{2}$ are generated by gauge and Yukawa interactions. The electro-weak vacuum $v \ll f$ is obtained by fine-tuning $C_{1}+C_{2} \approx v^{2} f^{2}$ to be small (i.e. by fine-tuning $g, g^{\prime}$ and fermionic mixings); the correct Higgs mass is obtained from a small $h^{4}$ term after including the SM RGE corrections between $f$ and $v$. We will come back to this point in the next section.

The field $a$ is now a good axion candidate. Indeed the right-handed up and down quarks are now charged under $\mathrm{U}(1)_{\mathrm{PQ}}$, with a QCD anomaly $N=2 N_{F}$, where $N_{F}=3$ is the number of generations. The electro-magnetic anomaly is,

$$
E=2\left[\left(\frac{4}{9}+\frac{1}{9}\right) 3+1\right] N_{F}+E_{\mathrm{TC}}
$$

where $E_{\mathrm{TC}}$ is the contribution of the TC fields. Therefore

$$
\frac{E}{N}=\frac{8}{3}+\frac{E_{\mathrm{TC}}}{6} .
$$

The model of eq. (4.3) predicts $E_{\mathrm{TC}}=2 n$ but this is not known in the model with partial compositeness since we ignore the microscopic theory. In general the electro-magnetic anomaly of the TC sector $E_{\mathrm{TC}}$ grows with the degrees of freedom of the TC sector, $E_{\mathrm{TC}} \propto n$, which could be large.

\section{The Higgs mass}

We now discuss the Higgs mass in $\mathrm{CH}$ models with large compositeness scale $\Lambda$. One motivation for such models, independent of axions, is that the Higgs quartic coupling $\lambda$, normalized such that $V(h)=m^{2} h^{2} / 2+\lambda h^{4} / 4$, is predicted to be small at $\Lambda$. Present data imply $\lambda\left(10^{11} \mathrm{GeV}\right)=-0.003 \pm 0.02$ (see figure $2 \mathrm{a}$ ). The leading contribution is of order

$$
\lambda(\Lambda) \sim g_{\mathrm{SM}}^{2} \frac{g_{\rho}^{2}}{(4 \pi)^{2}} \sim \text { few } 10^{-2}
$$

where $g_{\mathrm{SM}}$ indicates the various SM couplings $g, g^{\prime}, \lambda_{t}$ and $g_{\rho} \sim \Lambda / f$ is the coupling of the resonances of the strong sector, $1 \lesssim g_{\rho} \lesssim 4 \pi$. In a TC gauge theory $g_{\rho}$ is given by the large- $n$ relation $g_{\rho} \sim 4 \pi / \sqrt{n}$. In certain cases (see below) this dominant contribution to $\lambda$ cancels out, so that $\lambda$ is even smaller, generated only by SM interactions:

$$
\lambda(\Lambda) \sim \frac{g_{\mathrm{SM}}^{4}}{(4 \pi)^{2}} \sim 10^{-3}
$$

\footnotetext{
${ }^{8}$ Alternatively, one can assume that $\Psi_{U}$ forms a $\mathbf{1 0}$ of $\mathrm{SO}(6)$, which contains a single state with the quantum numbers of $u_{R}$, such that $\mathrm{U}(1)_{\mathrm{PQ}}$ is automatically conserved [27].
} 

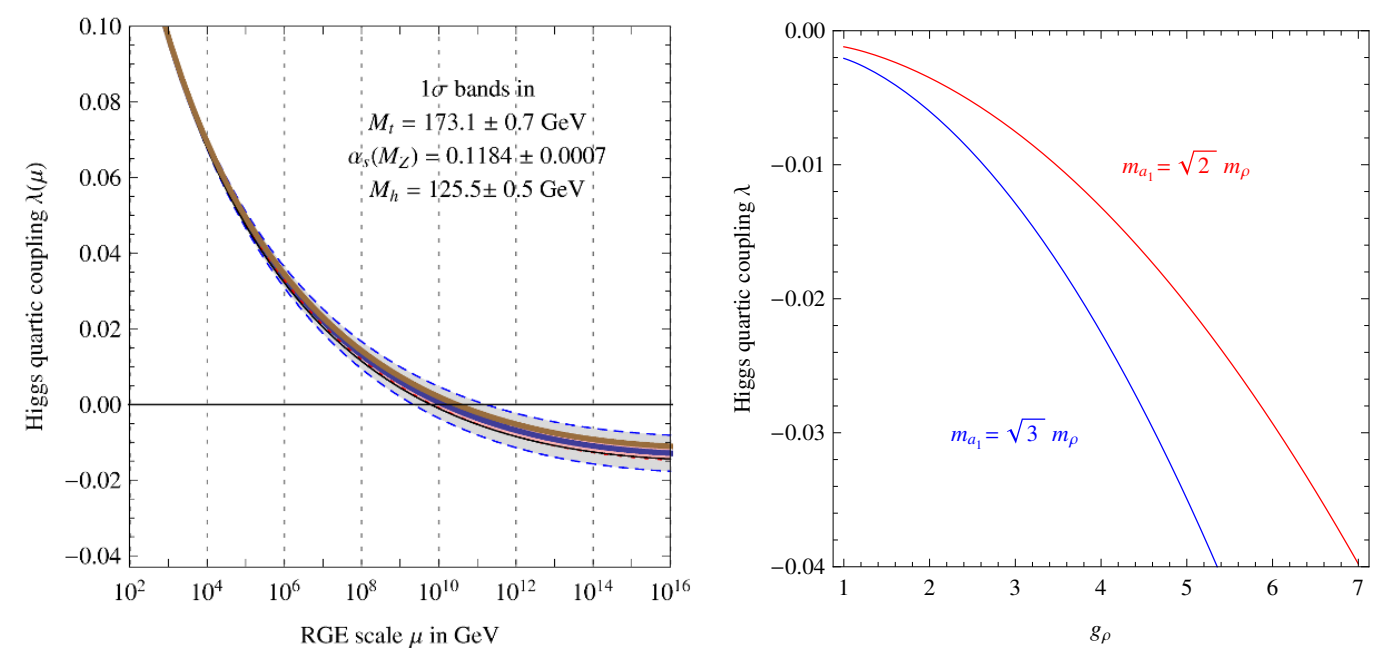

Figure 2. SM RGE evolution of the $\overline{M S}$ Higgs quartic coupling (left) and gauge contribution to the Higgs quartic coupling $\lambda$ renormalized at the compositeness scale, as a function of $g_{\rho}$ (right) for $m_{a_{1}}=\sqrt{2} m_{\rho}$ (upper curve) and $m_{a_{1}}=\sqrt{3} m_{\rho}$ (lower).

There are various contributions to the Higgs potential: SM gauge interactions and interactions that generate the SM fermion masses. Their sum must be tuned so the electroweak vacuum $v \ll f$ is generated. We start with the contribution of gauge fields that is essentially universal and can be reliably determined.

\subsection{The gauge contribution}

To be concrete we will frame our discussion for models where a single Higgs doublet is present.

The contribution to the effective potential of gauge loops is entirely analogous to the electro-magnetic splitting of pions in QCD and is given by the Coleman-Weinberg effective potential,

$$
V(h)_{\text {gauge }}=\frac{9}{2} \int \frac{d^{4} p}{(2 \pi)^{4}} \ln \left[1+F\left(p^{2}\right) \sin ^{2} \frac{h}{f}\right]
$$

where the form factor $F\left(p^{2}\right)$ is expressed in terms of the two point functions of the global currents of the theory, see [10] for more details. This can be computed explicitly assuming that the potential is dominated by the lowest resonances, like in QCD where they are called $\rho$ and $a_{1}$.

In models with low compositeness scale the momentum integral in eq. (5.3) is performed from zero to infinity. Since we want to take into account SM RGE effects between the weak scale and the compositeness scale, we prefer to estimate the boundary condition for $\lambda$ at the mass of the lowest resonances $\left(m_{\rho} \sim \Lambda\right)$. To do this we take a Wilsonian point of view and integrate over momenta between $m_{\rho}$ and infinity. Expanding the logarithm in eq. (5.3) 
at first order we obtain the leading corrections of eq. (5.1). More precisely following [31] we get

$$
\begin{aligned}
V(h)_{\text {gauge }} & \approx \frac{9}{2}\left(\int_{m_{\rho}}^{\infty} \frac{d^{4} p}{(2 \pi)^{4}} F\left(p^{2}\right)\right) \sin ^{2} \frac{h}{f} \\
& =\frac{9}{4} \frac{1}{16 \pi^{2}} \frac{g_{0}^{2}}{g_{\rho}^{2}} \frac{m_{\rho}^{4}\left(m_{a_{1}}^{2}-m_{\rho}^{2}\right)}{m_{a_{1}}^{2}-m_{\rho}^{2}\left(1+g_{0}^{2} / g_{\rho}^{2}\right)} \ln \left[\frac{m_{\rho}^{2}+m_{a_{1}}^{2}}{m_{\rho}^{2}\left(2+g_{0}^{2} / g_{\rho}^{2}\right)}\right] \sin ^{2} \frac{h}{f}
\end{aligned}
$$

where $m_{\rho}=g_{\rho} f / \sqrt{2} ; a_{1}$ are the vectors in $G / H$ that re-establish the global $G$ symmetry and thereby act as an UV cut-off; $g_{0}$ and $g_{\rho}$ are the elementary and composite couplings related to the SM couplings by,

$$
\frac{1}{g^{2}}=\frac{1}{g_{0}^{2}}+\frac{1}{g_{\rho}^{2}}
$$

The formula above includes the effect of mixing with elementary fields that may be relevant in the small $g_{\rho}$ region. The difference with respect to the integration from 0 to $\infty$ is contained in the logarithm, see [31]. Numerically for $m_{\rho}=10^{11} \mathrm{GeV}$ the typical reduction is by a factor $\sim 1.7$. To gain some intuition into this formula we take $m_{a_{1}}=\sqrt{2} m_{\rho}$ similarly to QCD, finding

$$
\lambda\left(m_{\rho}\right)_{\text {gauge }}^{\text {leading }} \approx-3 g_{0}^{2} \log \frac{3}{2} \frac{g_{\rho}^{2}}{(4 \pi)^{2}} .
$$

Using the full expression (5.4) one finds the result in figure 2. A quartic of the order of the experimental uncertainty requires $g_{\rho} \lesssim 3$. Note that the gauge contribution to the Higgs quartic is negative at the scale $m_{\rho}$ : this does not mean that the full quartic will be negative since other contributions to the potential must exist to tune the Higgs mass term to be $m \sim v$ rather than $m \sim f$. Indeed the gauge contribution to the Higgs mass term is given by

$$
\left.m^{2}\right|_{\text {gauge }} ^{\text {leading }}=-\frac{4}{3} f^{2} \lambda_{\text {gauge }}^{\text {leading }} .
$$

The TC coupling is expected to be of order $g_{\rho} \sim 4 \pi / \sqrt{n}$ for an $\mathrm{SU}(n)_{\mathrm{TC}}$ group, and thereby $g_{\rho}$ is small if $n$ is large - but not smaller than $g$, in view of eq. (5.5). In the examples in section 3 and 4 the measurable ratio $E / N$ grows with $n$.

\subsection{The fermion contribution and tuning}

The second unavoidable contribution to the potential is associated to the fermion couplings. This is model dependent: the SM fermions can couple linearly or bi-linearly to the strong sector fields whose quantum numbers under the global symmetries are also not known in general.

In composite models at the weak scale, the fermionic contribution is often dominant and the cancellation needed to obtain the electro-weak scale is between different fermionic contributions. This is no longer necessary in our case because at $\Lambda \approx 10^{11} \mathrm{GeV}$ the top Yukawa couplings is numerically similar to the weak gauge coupling, producing contributions to $\mathrm{m}^{2}$ and $\lambda$ of similar size as the gauge contributions. Therefore the weak scale 
can emerge as a huge cancellation in $m^{2}=m_{\text {gauge }}^{2}+m_{\text {fermions. Given the couplings that }}^{2}$ break the global symmetry of the theory, one could in principle compute whether such cancellation occurs for the observed values of the SM gauge and top Yukawa couplings.

If the electro-weak scale emerges through the cancellation of two leading order terms with different functional dependence on the Higgs (as in eq. (4.7)) the estimate of the quartic is given by eq. (5.1). This is the scenario typically considered in models with low compositeness scale. In our context a small quartic then requires a not very large TC coupling $g_{\rho}$.

Another possibility arises: in various models the leading order terms $V(h)_{\text {gauge }}$ and $V(h)_{\text {fermions }}$ have the same dependence on $h: \sin ^{2} h / f$. The cancellation of the $m^{2} h^{2} / 2$ term then implies a cancellation of the whole potential $V(h)$ at leading order in $g_{\rho}^{2}$. This is a problem in the context of the usual weak-scale compositeness models, where sub-leading terms are typically too small to reproduce the observed Higgs mass. Moreover this increases the tuning of the theory.

Having abandoned naturalness this cancelation becomes a virtue: it just implies that $\lambda \approx g_{\mathrm{SM}}^{4} /(4 \pi)^{2}$ at the compositeness scale. A non zero $\lambda$ at low energies is then generated by RGE running down to the weak scale, resulting in a Higgs mass of about $125 \mathrm{GeV}$. This happens for example in the model of section 2: $V(h)_{\text {fermions }}$ is considered in the appendix, finding that it is proportional to $\sin ^{2} h / f$, like the gauge contribution. This feature persists at higher orders in $g_{\rho}$.

\section{Conclusions and future directions}

We started to explore models where TC dynamics at some large scale $\Lambda$ spontaneously breaks a global symmetry, producing the Higgs and the axion as composite NambuGoldstone bosons, thereby unifying the Higgs with the axion. For a scale $\Lambda \approx 10^{11} \mathrm{GeV}$ this scenario has various phenomenological virtues:

1. the axion solves the QCD $\theta$ problem and provides the observed DM abundance. Experiments such as ADMX [21] are reaching the sensitivity necessary to test axion DM in the Milky Way and to measure the $a \gamma \gamma$ coupling, predicted in each model;

2. the observed Higgs mass is generated via SM RGE effects from a small Higgs quartic coupling at the compositeness scale, provided that the Higgs mass term is fine-tuned to be of electroweak size. In many models such fine-tuning implies a cancellation in the quartic Higgs coupling at the TC scale, such that very small (of order $\lambda \sim$ $g_{\mathrm{SM}}^{4} /(4 \pi)^{2}$ ) rather than small (of order $g_{\mathrm{SM}}^{2} g_{\mathrm{TC}}^{2} /(4 \pi)^{2}$ );

3. deviations from the SM are suppressed by powers of $\Lambda$, the dominant effect being Majorana neutrino masses $m_{\nu} \sim v^{2} / \Lambda$.

This scenario predicts no observable deviations from the SM in current precision, flavour and collider experiments. Like it or not, this is precisely what experiments found so far. In this situation, many efforts have been devoted to imagining new physics that escaped detection, at the price of building contrived models without any known UV completion. 
Data support the alternative possibility, that no new physics exists at the weak scale, motivating scenarios like the one advocated in the present paper. Simple models that do not make use of elementary scalars could lead to indirectly testable predictions.

In the present context, the next logical step would be to search for a simple theory of the 4-fermion operators responsible of fermion masses. Given that all effects beyond the SM are suppressed by the large scale $\Lambda \sim 10^{11} \mathrm{GeV}$, one can add extra flavour gauge symmetries broken by the TC dynamics. The main idea for a complete theory of these operators is Extended TechniColor (ETC). ${ }^{9}$

The fine-tuning needed to achieve $v \ll f$ implies a relation between the SM gauge couplings and the Yukawa couplings. If a predictive model will be found and its strong dynamics will be computed, such relation would allow to indirectly test if the weak scale really arises in this way.

\section{Acknowledgments}

We are grateful to Gilberto Colangelo, Rogerio Rosenfeld, Francesco Sannino and Giovanni Villadoro for useful discussions. This work was supported by the ESF grant MTT8 and by SF0690030s09 project.

\section{A Structure of the potential}

We here discuss the structure of the potential in the models of section 2 and 3 , following the discussion in ref. [32].

\section{A.1 Potential in the $\mathrm{SU}(6)_{L} \otimes \mathrm{SU}(6)_{R} / \mathrm{SU}(6)_{L+R}$ model}

The NGBs are described by an $\mathrm{SU}(6)$ matrix $U$. The gauging of SM group generates a potential for charged states analogous to the electro-magnetic splitting of the pions. It can be estimated as,

$$
V_{\text {gauge }} \sim \frac{3}{16 \pi^{2}} \Lambda^{2} f^{2} \sum_{a} g_{a}^{2} \operatorname{Tr}\left[U T^{a} U^{\dagger} T^{a}\right]
$$

where $T^{a}$ are the SM gauge generators. This generates a positive contribution to the squared mass of all the charged states. By assumption only the Higgs will be however light due to cancellation with different contributions to the potential.

Let us now turn to the fermions. Adding 4-Fermi operators one obtains a top Yukawa term

$$
C_{t} \sum_{\alpha=1}^{2}\left(q_{L}^{\alpha} t_{R}^{c}\right)^{\dagger} \operatorname{Tr}\left[\Pi_{t}^{\alpha} \cdot U\right]+\text { h.c. }
$$

\footnotetext{
${ }^{9}$ However so far we have not found any $\mathrm{SU}(n)$ ETC group with a chiral anomaly-free representation (such that fermions are massless and the PQ symmetry automatically arises) that leads to Composite Higgs. We instead find possibly viable models where TC-chiral fermions presumably break an extension of the SM gauge group, but we do not know how to study their strong dynamics.
} 
where $C_{t} \sim \Lambda f^{2} / \Lambda_{t}^{2} \sim f \lambda_{t}$. The matrices $\Pi_{t}$ are determined by the combination that couples to the SM fermions in eq. (2.9), given by

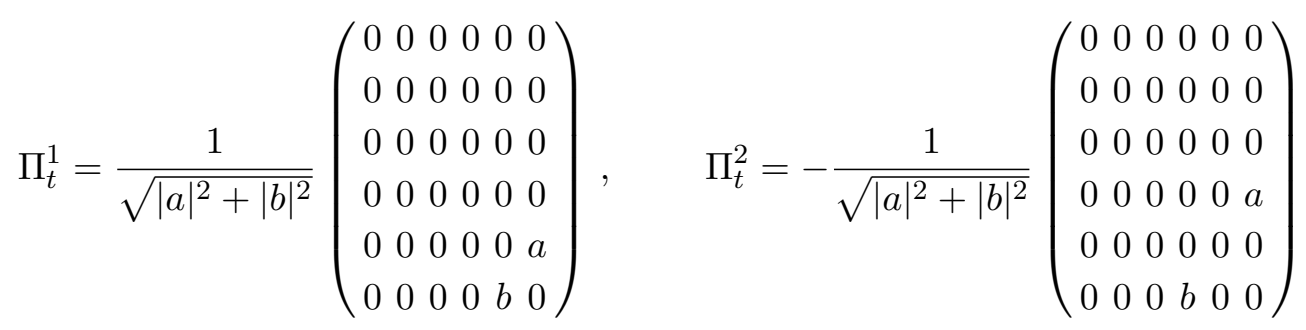

where the numbers $a, b$ depend on the specific 4 -fermion operator considered.

At 1-loop the leading contribution to the potential can be estimated as

$$
V_{\text {fermions }} \sim \frac{N_{c} \lambda_{t}^{2}}{16 \pi^{2}} \Lambda^{2} f^{2} \sum_{\alpha=1}^{2}\left|\operatorname{Tr}\left[\Pi_{t}^{\alpha} \cdot U\right]\right|^{2} .
$$

This term has a $\sin ^{2} h / f$ dependence on the Higgs field $h$, like the term in eq. (A.1) generated from gauge interactions.

\section{A.2 Potential in the $\mathrm{SU}(6) / \mathrm{SO}(6)$ model}

To describe the low energy interactions with this pattern it is useful to observe that the breaking can realized through the VEV of the symmetric representation of SU(6). The NGBs can be parametrized by

$$
\Phi=U \cdot \Phi_{0} \cdot U^{T}
$$

where $\Phi_{0}$ is any vacuum expectation value that breaks $\mathrm{SU}(6) \rightarrow \mathrm{SO}(6)$ (for example $\Phi_{0}=$ $\left.\operatorname{diag}\left(1,1,1,1, e^{i \alpha}, e^{-i \alpha}\right)\right)$ and $U=e^{i \pi^{\hat{a}} T^{\hat{a}} / f}$ where the sum extends to the broken generators. The low energy lagrangian is then simply obtained by writing formally $\mathrm{SU}(6)$ invariant terms containing $\Phi$.

The gauge contribution to the potential is now

$$
V_{\text {gauge }} \sim \frac{3}{16 \pi^{2}} \Lambda^{2} f^{2} \sum_{a} g_{a}^{2} \operatorname{Tr}\left[\Phi T^{a} \Phi^{\dagger} T^{a *}\right]
$$

with identical properties to the one discussed above. The 4-Fermi operators (3.5) generate

$$
\mathcal{L}=C_{t} \sum_{\alpha=1}^{2}\left(q_{L}^{\alpha} t_{R}^{c}\right)^{\dagger} \operatorname{Tr}\left[\Pi_{t}^{\alpha} \cdot \Phi\right]+C_{b} \sum_{\alpha=1}^{2}\left(q_{L}^{\alpha} b_{R}^{c}\right)^{\dagger} \operatorname{Tr}\left[\Pi_{b}^{\alpha} \cdot \Phi\right]+\text { h.c. }
$$

where the matrices associated to the top Yukawa are

$$
\Pi_{t}^{1}=\frac{1}{2}\left(\begin{array}{cccccc}
0 & 0 & 0 & 0 & 0 & 0 \\
0 & 0 & 0 & 0 & 0 & 0 \\
0 & 0 & 0 & 0 & 1 & i \\
0 & 0 & 0 & 0 & i & -1 \\
0 & 0 & 0 & 0 & 0 & 0 \\
0 & 0 & 0 & 0 & 0 & 0
\end{array}\right), \quad \Pi_{t}^{2}=\frac{1}{2}\left(\begin{array}{cccccc}
0 & 0 & 0 & 0 & 1 & i \\
0 & 0 & 0 & 0 & -i & 1 \\
0 & 0 & 0 & 0 & 0 & 0 \\
0 & 0 & 0 & 0 & 0 & 0 \\
0 & 0 & 0 & 0 & 0 & 0 \\
0 & 0 & 0 & 0 & 0 & 0
\end{array}\right)
$$


and similarly for the bottom sector.

The potential generated by quark loops at leading order is given by

$$
V_{\text {fermions }} \sim \frac{N_{c} \lambda_{t}^{2}}{16 \pi^{2}} \Lambda^{2} f^{2} \sum_{\alpha}\left|\operatorname{Tr}\left[\Pi_{t}^{\alpha} \cdot \Phi\right]\right|^{2}+\frac{N_{c} \lambda_{b}^{2}}{16 \pi^{2}} \Lambda^{2} f^{2} \sum_{\alpha}\left|\operatorname{Tr}\left[\Pi_{b}^{\alpha} \cdot \Phi\right]\right|^{2} .
$$

The bottom contribution could be relevant depending on $\tan \beta=v_{1} / v_{2}$. Expanding the top contribution around the field origin one finds

$$
V_{\text {fermions }} \sim \frac{N_{c} \lambda_{t}^{2}}{16 \pi^{2}} \Lambda^{2}\left(\left|H_{u}\right|^{2}+\frac{2 \sqrt{2}}{f} \operatorname{Im}\left[\sigma H_{u} H_{d}\right]+\ldots\right) .
$$

It is easy to see that the potential for $\sigma$ is controlled by the Higgs vacuum expectation value and is therefore suppressed in the electro-weak vacuum. This however is an accident of the one-loop approximation and at two-loop an unsuppressed potential for $\sigma$ will be generated that must be taken into account.

The singlet $\eta$ is an exact NGB as long as SM couplings are considered. This is phenomenologically allowed in view of $\Lambda \approx 10^{11} \mathrm{GeV}$. Anyhow, it can be made massive by adding to the Lagrangian mass terms for the TC-fermions that break explicitly the symmetry associated to $\eta$ and respect the SM gauge symmetry and the PQ symmetry:

$$
m_{L} L \bar{L}+m_{N} N \bar{N}+\text { h.c. }
$$

Similarly to quark masses in $\mathrm{QCD}$, this mass matrix $m$ generates

$$
V_{\text {mass }}=\Lambda f^{2} \operatorname{Tr}[m \cdot \Phi]+\text { h.c. } \simeq \Lambda m_{L}\left(12\left|H_{u}\right|^{2}+12\left|H_{d}\right|^{2}+\eta^{2}\right)+\Lambda m_{N}\left(6\left|H_{u}\right|^{2}+6\left|H_{d}\right|^{2}+6|\sigma|^{2}+\eta^{2}\right)+\ldots
$$

To realize the DFSZ axion model, $\sigma$ must spontaneously break the PQ symmetry getting a vacuum expectation value, which (in absence of tuning) is of order $f$. This corresponds to $\alpha \sim \pi / 2$ in eq. (A.5). Around this vacuum the Higgs potential must be tuned to reproduce the electro-weak vacuum $v \ll f$.

Open Access. This article is distributed under the terms of the Creative Commons Attribution License which permits any use, distribution and reproduction in any medium, provided the original author(s) and source are credited.

\section{References}

[1] ATLAS collaboration, G. Aad et al., Observation of a new particle in the search for the standard model Higgs boson with the ATLAS detector at the LHC, Phys. Lett. B 716 (2012) 1 [arXiv:1207.7214] [INSPIRE].

[2] CMS collaboration, S. Chatrchyan et al., Observation of a new boson at a mass of $125 \mathrm{GeV}$ with the CMS experiment at the LHC, Phys. Lett. B 716 (2012) 30 [arXiv:1207.7235] [INSPIRE].

[3] G. Degrassi, S. Di Vita, J. Elias-Miro, J.R. Espinosa, G.F. Giudice, et al., Higgs mass and vacuum stability in the standard model at NNLO, JHEP 08 (2012) 098 [arXiv:1205.6497] [INSPIRE]. 
[4] N. Arkani-Hamed and S. Dimopoulos, Supersymmetric unification without low energy supersymmetry and signatures for fine-tuning at the LHC, JHEP 06 (2005) 073 [hep-th/0405159] [INSPIRE].

[5] P.J. Fox, D.E. Kaplan, E. Katz, E. Poppitz, V. Sanz, et al., Supersplit supersymmetry, hep-th/0503249 [INSPIRE].

[6] G.F. Giudice and A. Strumia, Probing high-scale and split supersymmetry with Higgs mass measurements, Nucl. Phys. B 858 (2012) 63 [arXiv:1108.6077] [INSPIRE].

[7] E. Farhi and L. Susskind, Technicolor, Phys. Rept. 74 (1981) 277 [inSPIRE].

[8] K. Lane, Two lectures on technicolor, hep-ph/0202255 [INSPIRE].

[9] C.T. Hill and E.H. Simmons, Strong dynamics and electroweak symmetry breaking, Phys. Rept. 381 (2003) 235 [Erratum ibid. 390 (2004) 553-554] [hep-ph/0203079] [INSPIRE].

[10] K. Agashe, R. Contino and A. Pomarol, The minimal composite Higgs model, Nucl. Phys. B 719 (2005) 165 [hep-ph/0412089] [INSPIRE].

[11] M.J. Dugan, H. Georgi and D.B. Kaplan, Anatomy of a composite Higgs model, Nucl. Phys. B 254 (1985) 299 [INSPIRE].

[12] H. Georgi and D.B. Kaplan, Composite Higgs and custodial SU(2), Phys. Lett. B 145 (1984) 216 [INSPIRE].

[13] V. Agrawal, S.M. Barr, J.F. Donoghue and D. Seckel, The anthropic principle and the mass scale of the standard model, Phys. Rev. D 57 (1998) 5480 [hep-ph/9707380] [INSPIRE].

[14] J. Preskill, M.B. Wise and F. Wilczek, Cosmology of the invisible axion, Phys. Lett. B 120 (1983) 127 [INSPIRE].

[15] L. Abbott and P. Sikivie, A cosmological bound on the invisible axion, Phys. Lett. B 120 (1983) 133 [INSPIRE].

[16] M. Dine and W. Fischler, The not so harmless axion, Phys. Lett. B 120 (1983) 137 [INSPIRE].

[17] J.E. Kim, Weak interaction singlet and strong CP invariance, Phys. Rev. Lett. 43 (1979) 103 [INSPIRE].

[18] M.A. Shifman, A. Vainshtein and V.I. Zakharov, Can confinement ensure natural CP invariance of strong interactions?, Nucl. Phys. B 166 (1980) 493 [INSPIRE].

[19] A. Zhitnitsky, On possible suppression of the axion hadron interactions. (In Russian), Sov. J. Nucl. Phys. 31 (1980) 260 [INSPIRE].

[20] M. Dine, W. Fischler and M. Srednicki, A simple solution to the strong CP problem with a harmless axion, Phys. Lett. B 104 (1981) 199 [InSPIRE].

[21] ADMX collaboration, S. Asztalos et al., A SQUID-based microwave cavity search for dark-matter axions, Phys. Rev. Lett. 104 (2010) 041301 [arXiv:0910.5914] [INSPIRE].

[22] A. Arvanitaki, S. Dimopoulos, S. Dubovsky, N. Kaloper and J. March-Russell, String axiverse, Phys. Rev. D 81 (2010) 123530 [arXiv:0905.4720] [INSPIRE].

[23] S. Hsu and F. Sannino, New solutions to the strong CP problem, Phys. Lett. B 605 (2005) 369 [hep-ph/0408319] [INSPIRE].

[24] E. Farhi and L. Susskind, A technicolored G.U.T., Phys. Rev. D 20 (1979) 3404 [InSPIRE]. 
[25] G. Giudice, R. Rattazzi and A. Strumia, Unificaxion, Phys. Lett. B 715 (2012) 142 [arXiv: 1204.5465] [INSPIRE].

[26] E. Witten, Current algebra, baryons and quark confinement, Nucl. Phys. B 223 (1983) 433 [INSPIRE].

[27] B. Gripaios, A. Pomarol, F. Riva and J. Serra, Beyond the minimal composite Higgs model, JHEP 04 (2009) 070 [arXiv:0902.1483] [INSPIRE].

[28] D.B. Kaplan and H. Georgi, $\mathrm{SU}(2) \times U(1)$ breaking by vacuum misalignment, Phys. Lett. B 136 (1984) 183 [INSPIRE].

[29] D.B. Kaplan, H. Georgi and S. Dimopoulos, Composite Higgs scalars, Phys. Lett. B 136 (1984) 187 [INSPIRE].

[30] M. Redi and A. Tesi, Implications of a light Higgs in composite models, JHEP 10 (2012) 166 [arXiv: 1205. 0232] [INSPIRE].

[31] S. De Curtis, M. Redi and A. Tesi, The 4d composite Higgs, JHEP 04 (2012) 042 [arXiv:1110.1613] [INSPIRE].

[32] J. Galloway, J.A. Evans, M.A. Luty and R.A. Tacchi, Minimal conformal technicolor and precision electroweak tests, JHEP 10 (2010) 086 [arXiv:1001.1361] [INSPIRE]. 